\title{
NEW METHODS FOR HANDLING THE RANGE DEPENDENCE OF THE CLUTTER SPECTRUM IN NON-SIDELOOKING MONOSTATIC STAP RADARS
}

\author{
Fabian D. Lapierre (Research Fellow), Marc Van Droogenbroeck and Jacques G. Verly \\ University of Liège, Department of Electrical Engineering and Computer Science \\ Sart-Tilman, Building B28, B-4000 Liège, Belgium \\ \{ F.lapierre, M.VanDroogenbroeck, Jacques.Verly\}@ulg.ac.be
}

(C) IEEE 2002. Published in the 2003 International Conference on Acoustics, Speech, and Signal Processing (ICASSP 2003), scheduled for April 6-10, 2003 in Hong Kong SAR, China. Personal use of this material is permitted. However, permission to reprint/republish this material for advertising or promotional purposes or for creating new collective works for resale or redistribution to servers or lists, or to reuse any copyrighted component of this work in other works, must be obtained from the IEEE. Contact: Manager, Copyrights and Permissions / IEEE Service Center / 445 Hoes Lane / P.O. Box 1331 / Piscataway, NJ 08855-1331, USA. Telephone: + Intl. 908-562-3966.

\section{ABSTRACT}

We address the problem of detecting slow-moving targets using a non-sideloking monostatic space-time adaptive processing (STAP) radar. The construction of optimum weights at each range implies the estimation of the clutter covariance matrix. This is typically done by straight averaging of neighboring data snapshots. The range-dependence of these snapshots generally results in poor performance. We present two new methods that handle the rangedependence by exploiting the geometry of the direction-Doppler curves.

\section{INTRODUCTION}

Space-time adaptive processing (STAP) radars are used to detect slow-moving targets [1]. STAP relies on the transmission of a train of coherent pulses, the echos of which are received on a linear array-antenna. In monostatic (MS) radar configurations, the transmitter and the receiver are colocated. In sidelooking (SL) configurations, the antenna is parallel to the radar velocity vector. Prior research has mostly focused on SL MS configurations [1,2]. Here, we consider non-SL MS configurations.

The construction of the adaptive weights used for the optimal rejection of clutter at any given range implies the estimation of a clutter-plus-noise covariance matrix using data at neighboring ranges. In STAP, clutter is best described in terms of a $2 \mathrm{D}$ power spectral density (PSD) showing the distribution of expected power as a function of spatial and Doppler frequencies. These maps exhibit a clutter ridge, the shape of which changes with changing range for all non-SL MS configurations. This range-dependence creates major problems in the estimation of the covariance matrix.

Two approaches have been proposed so far to deal with this rangedependence. The "Doppler warping" method [3] works well in
nearly-SL MS configurations. The "scaling method" [4] works fairly well in all non-SL MS configurations, but can only exploit data at ranges greater than the range of interest. These methods are sensitive to uncertainties on the antenna crab angle $\delta$. The new methods work for all ranges. The first assumes that $\delta$ is known. The second works even if $\delta$ is unknown.

\section{MONOSTATIC GEOMETRY}

Figure 1 shows a canonical MS configuration, with a radar $R$ (typically airborne or spaceborne) and a scatterer $S$ (target or clutter patch). The origin of the coordinate system $(x, y, z)$ is chosen to coincide with $R$. The $x$-axis is aligned with the radar velocity vector $\underline{\boldsymbol{v}}_{R}$ and the $z$-axis points vertically up. The linear array-antenna is located in the $(x, y)$-plane and makes an angle $\delta$ with respect to the $x$-axis. $S$ is located at "cone" angles $\xi_{d}$ and $\xi$ with respect to the $x$-axis and the antenna axis, respectively. The range $R_{s}$ is the distance between $R$ and $S$.

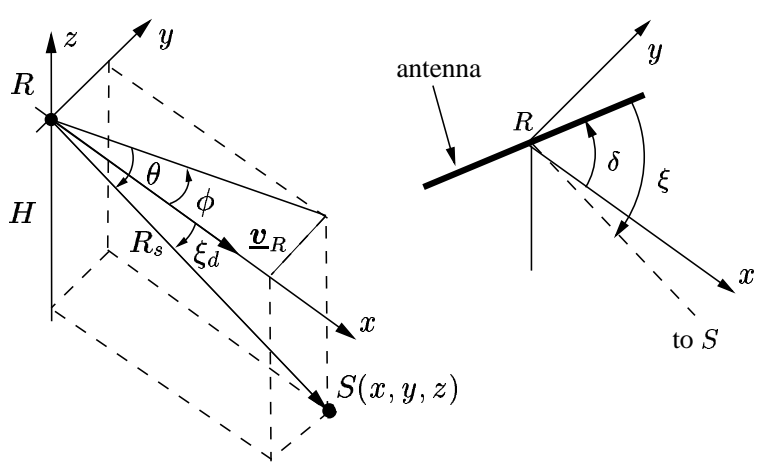

Fig. 1. Elements of a canonical MS radar configuration.

\section{DIRECTION-DOPPLER (DD) CURVES}

A radar should provide at least three parameters for each scatterer of interest: the angular position $\xi$, the range $R_{s}$ and the relative velocity $v_{r}$. These parameters can be computed from three other parameters that can be extracted from the radar returns: (1) The spatial frequency of the wave pattern along the antenna, $f_{s}=\cos \xi / \lambda_{c}$, where $\lambda_{c}$ is the carrier wavelength; (2) The 
roundtrip delay, $\tau=2 R_{s} / c$, where $c$ is the speed of the light; (3) The Doppler frequency, $f_{d}$, which for a stationary scatterer (such as clutter) is given by $f_{d}=2 v_{R} \cos \xi_{d} / \lambda_{c}$, where $v_{R}$ is the (signed) speed of $R$ along the $x$-axis.

In STAP, it is instructive to map all stationary scatterers at a given $R_{s}$ on an $\left(f_{s}, f_{d}\right)$ graph. The resulting locus is called a "direction-Doppler (DD)" curve. The graph's axes are typically labelled in terms of the normalized spatial and Doppler frequencies $\nu_{s}=\left(\lambda_{c} / 2\right) f_{s}$ and $\nu_{d}=\left(\lambda_{c} / 4 v_{R}\right) f_{d}$. Figure 2 shows a number of such graphs, each corresponding to a different $\delta$. Within each graph in Fig. 2, each curve corresponds to a different $R_{s}$. Note that the DD curves are range-dependent for all MS configurations other than SL $(\delta=0)$. The only other parameter influencing the shape of the curves is $v_{R}$. The parameter that runs along each curve is the angle $\phi$ of Fig. 1.
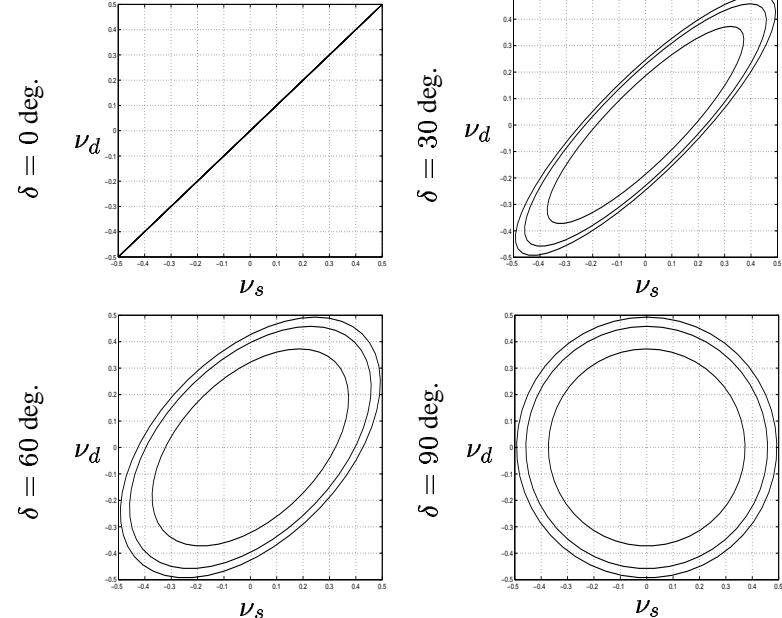

Fig. 2. Example DD curves for different combinations of crab angles $\delta$ and ranges $R_{s}(15,25$ and $50 \mathrm{~km}) ; v_{R}=90 \mathrm{~m} / \mathrm{s}$.

\section{OPTIMUM PROCESSOR}

$R$ transmits $M$ coherent pulses. The signals received at each of $N$ antenna-array elements are sampled, for each of the $M$ pulses, at a series of discrete ranges, called range gates. We treat these samples in space, time and range as a sequence in range of $M \times N$ data arrays called "snapshots". Each $M \times N$ snapshot corresponding to a single scatterer (target or clutter patch) with parameters $\nu_{s}, \nu_{d}$ and $R_{s}$ can be expressed as an $M N \times 1$ vector [2]

$$
\underline{\boldsymbol{y}}\left(\nu_{s}, \nu_{d}\right)=\beta_{r} \underline{\boldsymbol{v}}\left(\nu_{s}, \nu_{d}\right)
$$

where $\beta_{r}$ is a factor obtained from the radar equation and $\underline{\boldsymbol{v}}\left(\nu_{s}, \nu_{d}\right)$ is the $M N \times 1$ steering vector

$$
\underline{\boldsymbol{v}}\left(\nu_{s}, \nu_{d}\right)=\underline{\boldsymbol{b}}\left(\nu_{d}\right) \otimes \underline{\boldsymbol{a}}\left(\nu_{s}\right),
$$

where $\otimes$ is the Kronecker product and $\underline{\boldsymbol{a}}\left(\nu_{s}\right)$ and $\underline{\boldsymbol{b}}\left(\nu_{d}\right)$ are the $N \times 1$ spatial and $M \times 1$ temporal steering vectors given by

$$
\begin{aligned}
& \underline{\boldsymbol{a}}\left(\nu_{s}\right)=\left(1 \ldots e^{j 2 \pi \nu_{s} n} \ldots e^{j 2 \pi \nu_{s}(N-1)}\right)^{T} \\
& \underline{\boldsymbol{b}}\left(\nu_{d}\right)=\left(1 \ldots e^{j 2 \pi \nu_{d} m} \ldots e^{j 2 \pi \nu_{d}(M-1)}\right)^{T} .
\end{aligned}
$$

The $M N \times 1$ clutter snapshot $\underline{\boldsymbol{y}}_{c}\left(\nu_{s}, \nu_{d}\right)$ is found by integrating $\underline{\boldsymbol{y}}\left(\nu_{s}, \nu_{d}\right)$ over the isorange curve defined by the intersection, parameterized by $\phi$, of the isorange sphere with the ground,

$$
\underline{\boldsymbol{y}}_{c}\left(\nu_{s}, \nu_{d}\right)=\int_{0}^{2 \pi} \beta_{c}(\phi) \underline{\boldsymbol{v}}\left(\nu_{s}(\phi), \nu_{d}(\phi)\right) d \phi
$$

Since $\beta_{c}(\phi)$ is a random process, $\boldsymbol{y}_{c}$ is a random vector. We assume it is stationary. To find the power spectral density (PSD) associated with $\boldsymbol{y}$, we use spectral estimation methods. The minimum variance estimator (MVE) works well in STAP [1]. Clutter PSDs show a concentration of energy along a particular curve in the PSD array. The support of this "clutter ridge" is in direct correspondence with the related DD curve.

The weights of the optimum processor $(\mathrm{OP})$ providing optimum clutter rejection are given by the $M N \times 1$ vector [5]

$$
\underline{\boldsymbol{w}}_{\mathrm{opt}}\left(\nu_{s}, \nu_{d}\right)=\underline{\underline{\boldsymbol{R}}}^{-1} \underline{\boldsymbol{v}}\left(\nu_{s}, \nu_{d}\right)
$$

where $\underline{\underline{\boldsymbol{R}}}=E\left\{\underline{\boldsymbol{y}} \underline{\boldsymbol{y}}^{\dagger}\right\}$ is the sum of the covariance matrices $\underline{\underline{\boldsymbol{R}}}_{c}=$ $E\left\{\underline{\boldsymbol{y}}_{c} \underline{\boldsymbol{y}}_{c}^{\dagger}\right\}$ for the clutter and $\underline{\underline{\boldsymbol{R}}}_{n}=E\left\{\underline{\boldsymbol{y}}_{n} \underline{\boldsymbol{y}}_{n}^{\dagger}\right\}=\underline{\underline{\boldsymbol{I}}}$ for the noise, assumed to be spatially and temporally white. (Jammers are not considered here.) In practice, $\underline{\boldsymbol{R}}$ must be estimated for each $R_{s}$. The maximum-likelihood estimator $\underline{\underline{\widehat{\boldsymbol{R}}}}$ for range (gate) $l$ is [6]

$$
\underline{\underline{\widehat{\boldsymbol{R}}}}(l)=\frac{1}{N_{l}} \sum_{k \in S_{l}} \underline{\underline{\boldsymbol{R}}}(k) \quad \text { with } \quad \underline{\underline{\boldsymbol{R}}}(k)=\underline{\boldsymbol{y}}(k) \underline{\boldsymbol{y}}^{\dagger}(k)
$$

where $S_{l}$ is the set of surrounding-snapshots indices $k$ defined by $l-0.5\left(N_{l}-1\right)<k<l+0.5\left(N_{l}-1\right), N_{l}$ is the size of $S_{l}$ and $\boldsymbol{y}(k)$ and $\underline{\boldsymbol{R}}(k)$ the snapshot and the sample covariance matrix for range $k$. $\underline{\overline{\boldsymbol{R}}(l)}$ is unbiased only if the clutter ridge is range-independent. This happens only for SL configurations.

The performance of a processor using arbitrary weights $\underline{\boldsymbol{w}}$ is measured by the signal-to-interference-plus-noise ratio loss

$$
\operatorname{SINR}_{L}=\frac{\operatorname{SINR}}{\operatorname{SINR}_{0}}=\frac{|\underline{\boldsymbol{w}} \dagger \underline{v}|^{2}}{\left(\underline{\boldsymbol{w}}^{\dagger} \underline{\underline{\boldsymbol{R}}} \underline{\boldsymbol{w}}\right)\left(\underline{\boldsymbol{v}}^{\dagger} \underline{\boldsymbol{v}}\right)},
$$

where SINR $_{0}$ is the SINR in the absence of clutter. Values of SINR $_{L}$ range from the noise-to-clutter ratio to one. In practice, processor performance is degraded by estimation losses and by the fact that the $\underline{\underline{\boldsymbol{R}}}(k)$ 's in Eq. (5) are range-dependent. The goal of the new compensation methods described below is to eliminate or reduce the losses due to this range-dependence.

\section{NEW RANGE COMPENSATION METHODS}

The new methods replace Eq. (5) by

$$
\underline{\underline{\widehat{\boldsymbol{R}}}}(l)=\frac{1}{N_{l}} \sum_{k \in S_{l}} T_{k}[\underline{\underline{\boldsymbol{R}}}(k)],
$$

where the $T_{k}[$. ]'s are designed to compensate for the range-dependence and to approach the performance of the OP. 
Because of stationarity, $\underline{\underline{\boldsymbol{R}}}$ is Toeplitz-Block-Toeplitz and thus has redundant elements. It is thus possible to replace the $M N \times M N$ matrix $\underline{\boldsymbol{R}}$ by a $(2 N-1) \times(2 M-1)$ matrix $\underline{\boldsymbol{\Gamma}}$ entirely equivalent to $\underline{\underline{R}}$. In fact, $\underline{\underline{\Gamma}}$ is the matrix representation of the $2 \mathrm{D}$ autocorrelation function $\Gamma_{y y}$, where $y[n, m]$ is the $2 \mathrm{D}$ sequence representation of $\boldsymbol{y}$. Note that the dimensions of $\underline{\boldsymbol{\Gamma}}$ correspond to space and time. Focusing on $\underline{\underline{\boldsymbol{\Gamma}}}(k)$ 's with corresponding transformations $T_{k}^{\prime}[$. ], we have

$$
\widehat{\underline{\boldsymbol{\Gamma}}}(l)=\frac{1}{N_{l}} \sum_{k \in S_{l}} T_{k}^{\prime}[\underline{\underline{\Gamma}}(k)] .
$$

\subsection{Exact range-compensation (ERC) method}

The parameters $\underline{\boldsymbol{\theta}}$ influencing the shape of the DD curves are $v_{R}$, $\delta$ and $R_{s}$. Here, we assume that $v_{R}$ and $\delta$ are known. Of course, the various values of $R_{s}$ are also known, since we select the range gates. The design of $T_{k}^{\prime}[$.$] is based on the fact that all the non-SL$ DD curves for a given configuration are scaled versions of each other. In the exact range-compensation (ERC) method, we first determine, for each range $k$, the scaling transformation

$$
\left(\begin{array}{l}
\nu_{s}^{\prime}(k) \\
\nu_{d}^{\prime}(k)
\end{array}\right)=\left(\begin{array}{ll}
S_{s}(k) & 0 \\
0 & S_{d}(k)
\end{array}\right)\left(\begin{array}{l}
\nu_{s}(k) \\
\nu_{d}(k)
\end{array}\right)
$$

that scales the $\left(\nu_{s}, \nu_{d}\right)$-axes of the DD curve at range $k$ to bring it into registration with the DD-curve at the reference range $l$. Thus, the $\nu_{s}^{\prime}, \nu_{d}^{\prime}$ at range $k$ correspond exactly to the $\nu_{s}, \nu_{d}$ at range $l$. The scale factors in Eq. (6) can be determined exactly from the geometry. For a flat earth at height $H$, we find

$$
S_{s}(k)=S_{d}(k)=\sqrt{1-\left(H / R_{s}(l)\right)^{2}} / \sqrt{1-\left(H / R_{s}(k)\right)^{2}},
$$

where $R_{s}(i)$ is the true range corresponding to range gate $i$. The transformation is then applied, not to the DD curve plots, but to the corresponding PSDs. The main processing steps are shown in Fig. 3 and are now described.

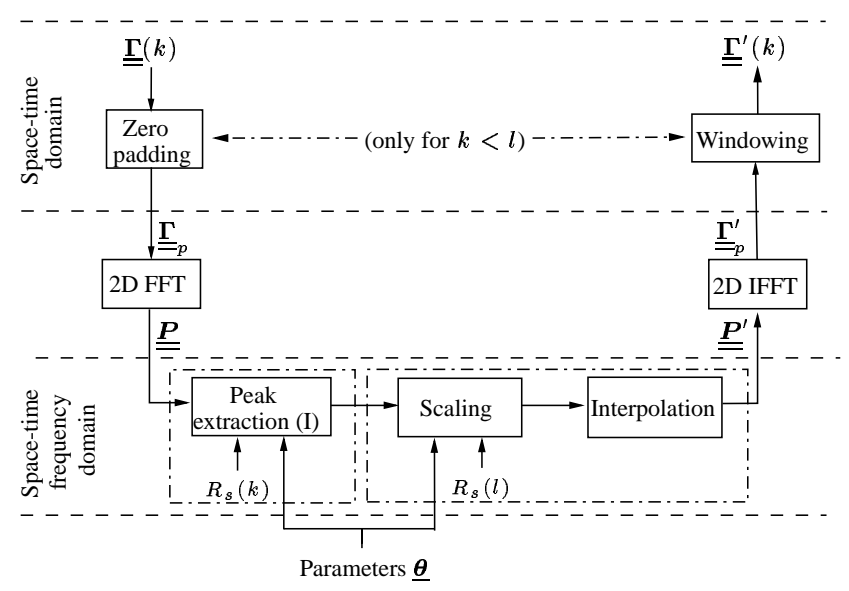

Fig. 3. Processing steps of the ERC method.

\subsubsection{Processing steps}

Zero padding (if required): Special care is required for ranges $k<l$. In these cases, the ultimate scaling of the PSD $\boldsymbol{P}$ is a dilation. This implies a contraction in the inverse Fourier domain, i.e., of $\underline{\underline{\boldsymbol{\Gamma}}}$. To allow for this contraction, we must increase the size $(2 N-1) \times(2 M-1)$ of $\underline{\underline{\Gamma}}$ by factors of $S_{s}$ and $S_{d}$ in the $\nu_{s}$ and $\nu_{d}$ dimensions, respectively. This expansion is performed using zero padding. Of course, no padding is done for $k>l$. The output is denoted by $\underline{\underline{\boldsymbol{\Gamma}}}_{p}$, whether it is padded or not.

\section{Fourier transform: The FFT of $\underline{\underline{\boldsymbol{\Gamma}}}_{p}$ gives the PSD $\underline{\underline{\boldsymbol{P}}}$.}

Peak extraction (I): Our goal is to dilate or contract (as required) the clutter ridge in $\underline{\boldsymbol{P}}$. To avoid scaling points that are outside the clutter ridge or $\overline{\overline{o n}}$ its sidelobes, we find the position of the significant peaks in $\underline{\boldsymbol{P}}$. We can easily track these peaks down along the theorical DD curve since the configuration is known exactly.

Scaling: First, we compute $S_{s}$ and $S_{d}$ according to Eq. (7). Then, we compute the new position $\left(\nu_{s}^{\prime}(k), \nu_{d}^{\prime}(k)\right)$ of the extracted peaks. The original peak intensities are assigned to the nearest pixels in $\underline{\underline{\boldsymbol{P}}}^{\prime}$.

Interpolation: When $k<l$, linear interpolation is performed to ensure the "continuity" of the dilated ridge in $\underline{\underline{\boldsymbol{P}}}^{\prime}$.

Inverse Fourier transform: The IFFT of the PSD $\underline{\underline{\boldsymbol{P}}}^{\prime}$ gives ${\underline{\underline{\boldsymbol{\Gamma}_{p}}}}_{p}$.

Windowing: If $k<l, \underline{\underline{\Gamma}}_{p}^{\prime}$ is larger by a factor $S_{s} \times S_{d}$ than the desired $\underline{\underline{\Gamma}}^{\prime}$. Thus, we must window $\underline{\underline{\Gamma}}_{p}^{\prime}$ to recover the desired $(2 N-1) \times(2 M-1) \underline{\underline{\Gamma}}^{\prime}$.

\subsubsection{Example}

The desired $\underline{\boldsymbol{R}}^{\prime}(k)$ can easily be reconstructed from $\underline{\underline{\Gamma}}^{\prime}(k)$. Figure 4 shows the MVE of $\underline{\boldsymbol{R}}(k)$ (before compensation) and the MVE of $\underline{\underline{\boldsymbol{R}}}^{\prime}(k)$ (after compensation).

PSD

before compensation

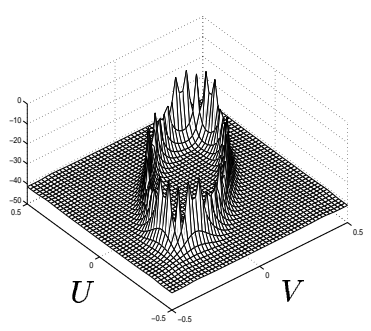

Fig. 4. Effect of ERC-designed transformation $T_{k}^{\prime}[$.$] .$

\subsection{Blind range-compensation (BRC) method}

In practice, we can assume that $v_{R}$ is known, in particular if the processing is done on $\underline{\boldsymbol{R}}$. However, the angle $\delta$ is not generally known with accuracy, especially in the presence of lateral wind and 
wind gusts. The blind range-compensation (BRC) method does not require that $\delta$ be known. The BRC method is build on top of the ERC method, as shown in Fig. 5. The new processing step is the estimation of the parameters $\underline{\boldsymbol{\theta}}$, i.e., $\delta$ in the present case. The new processing steps are as follows.

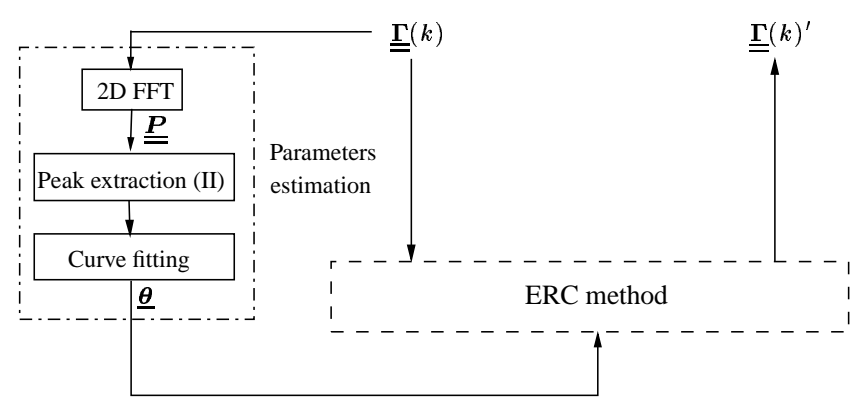

Parameters $\underline{\boldsymbol{\theta}}$

Fig. 5. Processing steps of the BRC method

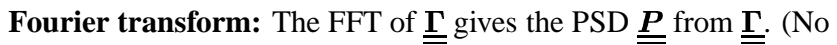
padding is performed here.)

Peak extraction (II): We need to find the most significant peaks in $\boldsymbol{P}$. However, since $\delta$ is not known initially, we cannot use the "peak extraction" algorithm of Fig. 3. Instead, we use a thresholding algorithm. This algorithm uses the histogram of the peak amplitudes to find the optimal threshold. Figure 6(b) shows a typical example of such a histogram. Not surprisingly, the histogram is bimodal. The high intensities (on the right) correspond to the ridge and the low intensities (on the left) to the rest of the values in $\underline{\underline{\boldsymbol{P}}}$, some of which correspond to the sidelobes of the FFT. To identify the peaks, we need to find the threshold that best separates the two lobes of the histogram. Here, this is done by taking the first bin for which the number of occurrences observed is less than five percent of the maximum number of occurences. More sophisticated algorithms could be used if necessary. The resulting thresholded Fourier transform is shown in Fig. 6(c).

Curve fitting: In MS configurations, $\delta$ is the only unknown parameter. However, the parameter estimation problem can be formulated in term of a general vector $\underline{\boldsymbol{\theta}}$ of unknown parameters. $\underline{\boldsymbol{\theta}}$ is found using the position of the detected peaks. This is a fitting problem: we have the equation of the parametric DD curve and a set of experimental points $\left(\nu_{s}(j), \nu_{d}(j)\right)$. The optimum value of $\underline{\boldsymbol{\theta}}$ is found by minimizing the MSE given by

$$
\sum_{j=1}^{N_{p}} d^{2}\left(\mathcal{C}(\underline{\boldsymbol{\theta}}),\left(\nu_{s}(j), \nu_{d}(j)\right)\right)
$$

where $N_{p}$ is the number of detected peaks, $d(\mathcal{C}, P)$ is the distance between curve $\mathcal{C}$ and point $P$. $\mathcal{C}(\underline{\theta})$ is the DD curve corresponding to $\underline{\boldsymbol{\theta}}$. Its equation is $\nu_{s}^{2}+\nu_{d}^{2}+-2 \nu_{s} \nu_{d} \cos \delta=$ $0.25 \sin ^{2} \delta\left(1-\left(H / R_{s}\right)^{2}\right)$. Using samples $\left(\nu_{s}(j), \nu_{d}(j)\right)$, we con-

struct an overdetermined system $\underline{\underline{\boldsymbol{A}}} \underline{\boldsymbol{x}}=\underline{\boldsymbol{b}}$, where

$$
\begin{aligned}
\underline{\underline{\boldsymbol{A}}} & =\left(\begin{array}{cc}
2 \nu_{s}(0) \nu_{d}(0) & \frac{1}{4}\left(1-\left(H / R_{s}\right)^{2}\right) \\
\vdots & \vdots \\
2 \nu_{s}\left(N_{p}-1\right) \nu_{d}\left(N_{p}-1\right) & \frac{1}{4}\left(1-\left(H / R_{s}\right)^{2}\right)
\end{array}\right), \\
\underline{\boldsymbol{b}} & =\left(\nu_{s}^{2}(0)+\nu_{d}^{2}(0) \ldots \nu_{s}^{2}\left(N_{p}-1\right)+\nu_{d}^{2}\left(N_{p}-1\right)\right)^{T}, \\
\underline{\boldsymbol{x}} & =\left(\cos \delta \sin ^{2} \delta\right)^{T} .
\end{aligned}
$$

The least-mean-square solution $\underline{\boldsymbol{x}}_{0}$ is

$$
\underline{\boldsymbol{x}}_{o}=\left(\underline{\underline{\boldsymbol{A}}}^{T} \underline{\underline{\boldsymbol{A}}}\right)^{-1} \underline{\underline{\boldsymbol{A}}}^{T} \underline{\underline{b}}
$$

Note that we have to treat $\cos \delta$ and $\sin ^{2} \delta$ as distinct unknowns. Among the two values of $\delta$,

$$
\delta_{1}=\arccos \left(\underline{\boldsymbol{x}}_{o}(0)\right) \text { and } \delta_{2}=\sqrt{\arcsin \left(\underline{\boldsymbol{x}}_{o}(1)\right)},
$$

we choose the one with the smallest residue.
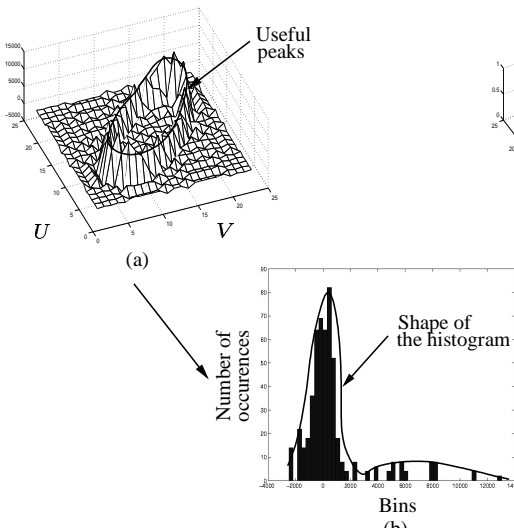

(b)

Fig. 6. Peak-extraction algorithm used for parameter estimation in the BRC method.

\section{PERFORMANCE COMPARAISON}

Figure 7 compares the performances of the ERC method to those of the straight averaging (SA) technique and the OP. ERC is much better than SA and nearly as good as OP. Fig. 7 also compares ERC to BRC (and OP). BRC is nearly as good as RC in this particular case. Similar performance is achieved with directive sensors.

\section{CONCLUSION}

We have proposed two new methods for compensating the range-dependence of PSDs in non-sidelooking monostatic STAP. Whereas the ERC method assumes that the configuration parameters are known, the BRC method estimates the unknown parameters ( $\delta$ in MS configurations) prior to applying the ERC method. The ERC and the BRC methods provide near optimal performance. 

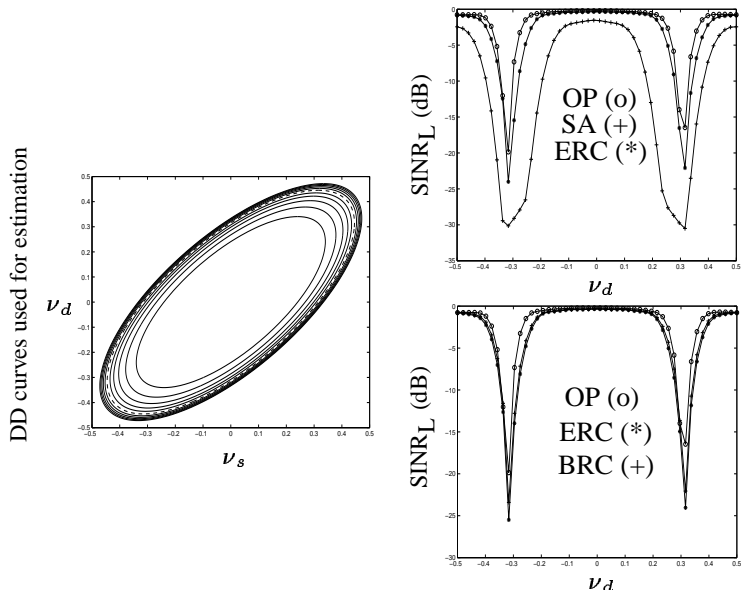

Fig. 7. Performance comparaison of ERC, BRC, SA and OP methods. Graphs show slice of the $\operatorname{SINR}_{\mathrm{L}}$ at $\nu_{s}=0$.

\section{REFERENCES}

[1] R. Klemm, Space-Time Adaptive Processing : Principles and Applications, IEE Radar, Sonar, Nav. and Avionics 9, 2000.

[2] J. Ward, "Space-time adaptive processing for airborne radar," Technical Report 1015, MIT Lincoln Laboratory, 1994.

[3] G.K. Borsari, "Mitigating effects on stap processing caused by an inclined array," IEEE National Radar Conference, Dallas, pp. 135-140, 12-13 May 1998.

[4] F.D. Lapierre and J.G. Verly, "The range-dependance problem of clutter spectrum for non-sidelooking monostatic stap radars," 21st Benelux Meeting on Systems and Control, Veldhoven, The Netherlands, March 2002.

[5] L.E. Brennan and L.S. Reed, "Theory of adaptive radar," IEEE Transactions on Aerospace and Electronic Systems (AES), vol. 9, no. 2, pp. 237-252, 1973.

[6] I.S. Reed, J.D. Mallett, and L.E. Brennan, "Rapid convergence rate in adaptive arrays," IEEE Trans. on Aerospace and Electronic Systems (AES), vol. 10, no. 6, pp. 853-863, 1974. 